\section{APERTURE FIELD RECONSTRUCTION BY CALCULATED CYLINDRICAL NEAR FIELD}

Alcino Castelo Boso, Leandro de Haro Ariet, and José Luis Besada

Departamento Sistemas, Señales y Radiocomunicaciones, ETSI

Telecomunicación, Universidad Politécnica de Madrid, Ciudad

Universitaria, Madrid 28040, Spain; Corresponding author:

\begin{abstract}
A method for determining the aperture field distribution of an array antenna from near-field measurement over the surface of a right circular cylinder enclosing the antenna is applied here. The approach relies on the concept of information content of the field. The truncation problem is solved by picking up the information that is lost due to the finite size of scanning area, in points of the space reachable by the measurement system. In this article, we rebuild the field at the aperture from near-field cylindrical calculated on the surface of measure, with dependence on the variable phi, and without dependence on the variable phi.
\end{abstract}

Key words: antenna measurement; aperture field; variable phi

\section{INTRODUCTION}

The method for determining the aperture field distribution of an antenna from near-field measurements over the surface of a right circular cylinder enclosing the antenna has been widely employed to determine the far-field patterns of antennas under test. This is derived from the wave modal expansion theory that has already been employed in near-field to far-field transformation techniques based on the three-dimensional vector cylindrical wave expansion of an electromagnetic field.

The truncation error is due to the finite size of the scanning surface, for the minimization of the error source, in the development of near-field measurements. The standard approach to reduce the truncation error is to extend the measurement surface up to include the entire region wherein the field radiated by the antenna under test is significant The a priori information consists in the overall dimension of the antenna, in its position in the space and in further information on the shape of the antenna.

It is possible to recover the information associated with the samples falling in the part of the scanning surface external to the actual scanning area by measuring the field at other points around the AUT that area reachable by the scanning set-up. The samples of the representation falling inside the actual scanning area are directly measured. To recover the external samples, measurements having the same information content of the lost samples are performed in an area reachable by the measurement set-up, usually in a frame around the available scanning area. Then, a linear system relating the samples on the ideal scanning surface and the data measured on the frame is implemented by means of aperture field reconstructions near-field transformation

In this article, we reconstruct the field at the aperture from near-field cylinder calculated on the surface of measure, with dependence on the variable phi, and without dependence on the variable phi.

\section{FORMULATION}

In a linear, isotropic region containing no sources, a solution $\psi_{n}^{i}$ to the scalar wave equation in the cylindrical coordinate system is

$$
\psi_{n}^{i}(r, \phi, z)=Z_{n}^{i}(\Lambda r) \exp (j n \phi) \exp (-j h z)
$$

where $n$ is any integer, $h$ is any real number, and $\Lambda$ is

$$
\Lambda=\left\{\begin{array}{cc}
\sqrt{k^{2}-h^{2}} & h \leq k \\
-j \sqrt{h^{2}-k^{2}} & h>k
\end{array}\right.
$$

The vectors $M_{n}$ and $N_{n}$ are written as

$$
\begin{array}{r}
M_{n h}^{i}(r, \phi, z)=\left(\hat{r}_{r} \frac{j n}{r} Z_{n}^{i}(\Lambda r)-\hat{\phi} \frac{\partial Z_{n}^{i}}{\partial r}\right) \exp (j n \phi) \exp (-j h z) \\
N_{n h}^{i}(r, \phi, z)=\left(-\hat{r} \frac{j h}{k} \frac{\partial Z_{n}^{i}}{\partial r}+\hat{\phi} \frac{n h}{k r} Z_{n}^{i}+\hat{z} \frac{\Lambda^{2}}{k} Z_{n i}^{i}(\Lambda r)\right) \exp (j n \phi) \exp \\
(-j h z)
\end{array}
$$

The function $Z_{n}^{i}(\Lambda r)$ is any one of the four cylindrical Bessel functions given by

$$
\begin{gathered}
Z_{n}^{1}(\Lambda r)=J_{n}(\Lambda r), Z_{n}^{2}(\Lambda r)=Y_{n}(\Lambda r), Z_{n}^{3}(\Lambda r)=H_{n}^{(1)}(\Lambda r), Z_{n}^{4}(\Lambda r) \\
=H_{n}^{(2)}(\Lambda r)
\end{gathered}
$$

In the present case, a solution for $E$ is required, which is valid in the region external to a small cylinder containing all sources defined by $r \geq r_{a}$. In order for $E$-field to satisfy the radiation condition at infinity, the large argument asymptotic expansion of the cylindrical Bessel function must represent surfaces of constant phase which propagates in the positive radial direction. For time variations of the form $\exp (j w t)$, the only one of these functions which satisfies this condition is a Hankel function of the second kind $H_{n}^{(2)}(\Lambda r)$ [2]. The large argument asymptotic expansion of this function is

$$
H_{n}^{(2)}(\Lambda r) \rightarrow j^{n+\frac{1}{2}} \sqrt{\frac{2}{\pi \Lambda r} \exp (-j \Lambda r)} ; r \rightarrow \infty
$$

The general linear combination of the vectors $M$ and $N$ will involve an integral over all real $h$ and a sum over all integer $n$. Thus, in this case, the general solution for $E$-field can be written as

$$
E(r, \phi, z)=\sum_{n=-\infty}^{\infty} \int_{-\infty}^{\infty}\left(a_{n}(h) M_{n l h}^{4}(r, \phi, z)+b_{n}(h) N_{n h}^{4}(r, \phi, z)\right) d h
$$

where $a_{n}(h)$ and $b_{n}(h)$ are the complex amplitude weighting functions of the vectors $M$ and $N$, respectively [2]. The corresponding solution for $H$ follows from Maxwell's equation

$$
\begin{gathered}
H=(-1 / j w \mu) \nabla \times E \text { and is given by } \\
H(r, \phi, z)=\frac{-k}{j w \mu} \sum_{-\infty}^{\infty} \int_{-\infty}^{\infty}\left(b_{n}(h) M_{n h}^{4}(r, \phi, z)+a_{n}(h) N_{n h}^{4}(r, \phi, z)\right) d h
\end{gathered}
$$

For magnetic and electric components can be written as 


$$
\begin{gathered}
H_{z}(r, \phi, z)=\frac{-1}{j w \mu} \sum_{n=-\infty}^{\infty} \int_{-\infty}^{\infty} \Lambda^{2} a_{n}(h) H n^{(2)}(\Lambda r) e^{j n \phi-j h z} d h \\
H_{\phi}(r, \phi, z)=\frac{1}{j w \mu} \sum_{n=-\infty}^{\infty} \int_{-\infty}^{\infty}\left(b_{n}(h) k\right. \\
\left.\times \frac{\partial H n^{(2)}(\partial r)}{\partial r}-a_{n}(h) \frac{n h}{r} H n^{(2)}(\Lambda r)\right) e^{j n \phi-j h z} d h \quad(9) \\
H_{r}(r, \phi, z)=\frac{1}{j w \mu} \sum_{n=-\infty}^{\infty} \int_{-\infty}^{\infty} \\
\times\left(-b_{n}(h) k \frac{j n k H n^{(2)}(\Lambda r)}{r}+a_{n}(h)(j h k) \frac{\partial H n^{(2)}(\Lambda r)}{\partial r}\right) e^{j n \phi-j h z} d h \\
E_{z}\left(r_{0}, \phi, z\right)=\sum_{n=-\infty}^{+\infty} \int_{-\infty}^{+\infty} b_{n}(h) \frac{\Lambda}{k} H_{n}^{(2)}\left(\Lambda r_{0}\right) e^{j n \phi-j h z} d h \\
E_{\phi}\left(r_{0}, \phi, z\right)=\sum_{n=-\infty}^{+\infty} \int_{-\infty}^{+\infty}\left[b_{n}(h) \frac{n h}{k r_{0}} H_{n}^{(2)}\left(\Lambda r_{0}\right)-a_{n}(h) \frac{\partial H_{n}^{(2)}\left(\Lambda r_{0}\right)}{\partial r}\right] \\
\times e^{j n \phi-j h z} d h \quad(12)
\end{gathered}
$$

and their inverse

$$
a_{n}(h)=\frac{-j \omega \mu}{\Phi^{2} H_{n}^{(2)}(\Phi r)} \int_{-\infty}^{\infty} \int_{-\pi}^{\pi} H_{z}(r, \phi, z) e^{-j n \phi} e^{j h z} d \phi d z
$$

$b_{n}(h)=\frac{k}{\Phi^{2} H n^{(2)}\left(\Phi r_{0}\right)} \int_{-\infty}^{\infty} \int_{-\pi}^{\pi} E_{z}\left(r_{0}, \phi, z\right) \exp (-j n \phi) \exp (j h z) d \phi d z$

$$
\begin{aligned}
b_{n}(h) \frac{n h}{k r_{0}} H_{n}^{(2)}\left(\Lambda r_{0}\right)- & a_{n}(h) \frac{\partial H_{n}^{(2)}\left(\Lambda r_{0}\right)}{\partial r} \\
& =\frac{1}{4 \pi^{2}} \int_{-\pi}^{\pi} \int_{-\infty}^{+\infty} E_{\phi}\left(r_{0}, \phi, z\right) e^{-j n \phi} e^{j h z} d \phi d z
\end{aligned}
$$

Let

$$
\int_{-\pi}^{\pi} \exp (-j n \phi) d \phi=\left\{\begin{array}{cc}
2 \pi & n=0 \\
0 & n \neq 0
\end{array}\right.
$$

then, $b_{n}$ becomes $b_{0}\left(b_{n}=0\right.$ for $\left.n \neq 0\right)$ only:

$$
b_{n}(h)=b_{0}(h)=\frac{2 \pi k}{\Lambda^{2} H o^{(2)}\left(\Lambda r_{0}\right)} \int_{-\infty}^{\infty} E_{z}\left(r_{0}, \phi, z\right) \exp (j h z) d z
$$

Since the $z$ component of the magnetic field, $H_{z}\left(r_{0}, \phi, z\right)$, is zero in this cases, it follows from, (13) that the other amplitude weighting function $a_{0}(h)$ is correspondingly zero. Thus the normalized fields radiated by the dipole or array of dipoles on any cylinder can be expressed in terms of $b_{0}(h)$ as

$$
E_{z}\left(r_{1}, \phi_{1}, z_{1}\right)=\frac{2 \pi k H_{0}^{(2)}(\Lambda r)}{H_{0}^{(2)}\left(\Lambda r_{0}\right)} \int_{-\infty}^{\infty} \int_{-\infty}^{\infty} E_{z}\left(r_{0}, \phi, z\right) d z d h
$$

and

$$
E_{\phi}\left(r_{1}, \phi_{1}, z_{1}\right)=\frac{4 \pi^{2} k u h H_{0}^{(2)}(\Lambda r)}{\Lambda^{2} H_{0}^{(2)}\left(\Lambda r_{0}\right) k r_{0}} \int_{-\infty}^{\infty} \int_{-\infty}^{\infty} E_{z}\left(r_{0}, \phi, z\right) d z d h
$$

$u$ is an integer different of zero.

Whenever, if the $z$ component of $E$-field radiated by the centerfed dipole of length $L$ with sinusoidal current distribution is

$$
\begin{gathered}
E_{z}=\frac{-j I_{0} \eta}{4 \pi}\left(\frac{e^{-j k s 1}}{S_{1}}+\frac{e^{-j k s s_{2}}}{S_{2}}-2 \cos \left(\frac{k L}{2}\right) \frac{e^{-j k R}}{R}\right) \\
R=\sqrt{r^{2}+z^{2}} ; s_{1}=\sqrt{r^{2}+\left(z-\frac{L}{2}\right)^{2}} ; s_{2}=\sqrt{r^{2}+\left(z+\frac{L}{2}\right)^{2}}
\end{gathered}
$$

where $k=2 \pi / \lambda$ is the wavenumber, $\eta$ is intrinsic impedance of free space and $I_{0}$ is maximum current, the $z$ component of $E$-field radiated [Eq. (20)] by the center-fed dipole and array of dipoles linearly along the axis $z$, is independent of $\phi$.

\section{DISCUSSION AND NUMERICAL EXAMPLES}

In this section, we report some numerical examples assessing the effectiveness of the work; The AUT consists of a linear array of $3 \times 25$ dipoles oriented along the $z$ axis, with equal excitation, and uniformly spaced on a square lying in the $x z$ plane and $\lambda / 2$ equispaced. Each dipole is fed by a unit electric current. The near-field component along $\hat{z}$ of the electric field is measured by a probe on a scanning surface placed at a distance $d=54 \lambda$ from the AUT. The reconstruction has been made considering 153 points along $\phi$ and 145 points along the $z$ axis on the surface of a

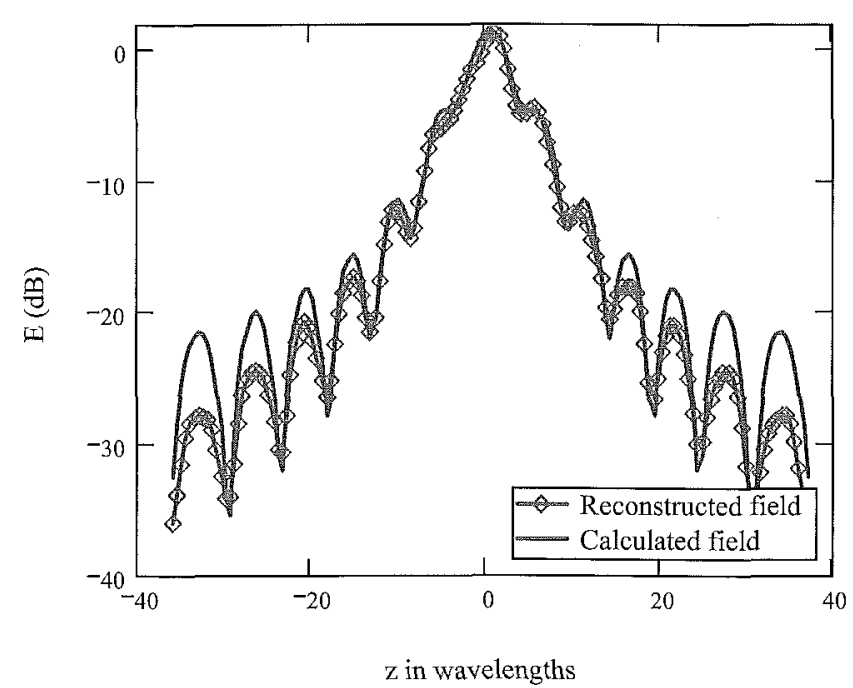

Figure 1 Reconstructed and calculated field $\left(E_{z}\right)$ with dependence of the component phi. 


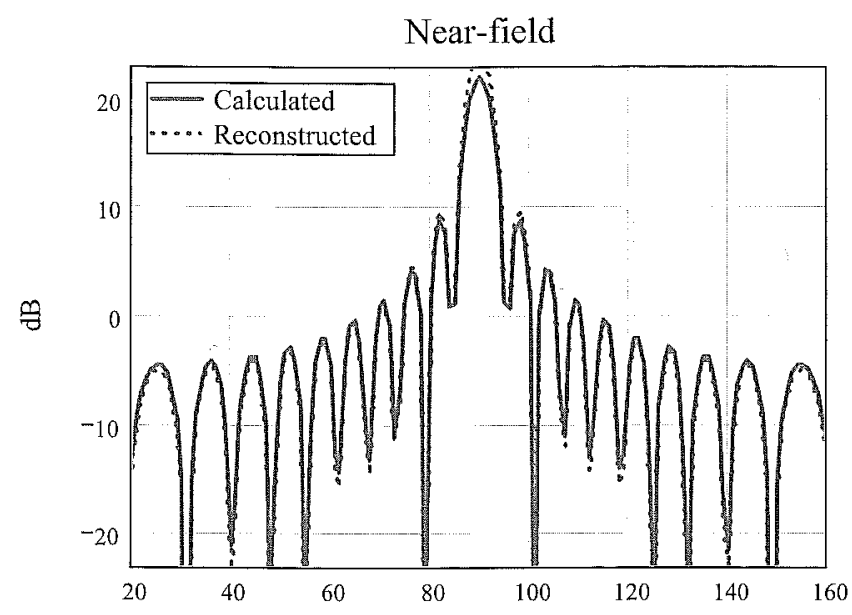

Theta in degree

Figure 2 Calculated and reconstructed field $\left(E_{7}\right)$ of array dipoles without dependence of the component phi.

cylinder, and the practical upper bounds on the sample intervals are $\Delta \phi=\frac{2 \pi}{N}$ and $\Delta z=\frac{z_{\max }}{M}$, and a dipole and array of dipoles of length $L$ with sinusoidal current distribution, linearly along the axis $z$.

Then we will see diagrams of radiation in the near-field component $z$, calculated and reconstructed, for an array of dipoles and for a dipole, with dependence and independence of the variable phi.

We see in Figure 1 diagram of the near-field calculated and reconstructed for an array of dipoles distributed rectangular taking into account the electric field component phi. While in Figure 2 we see the near-field diagram calculated and reconstructed for an array of dipoles distributed linearly therefore not taking into account the electric field component phi.

We see in Figure 3 diagram of the near-field calculated and

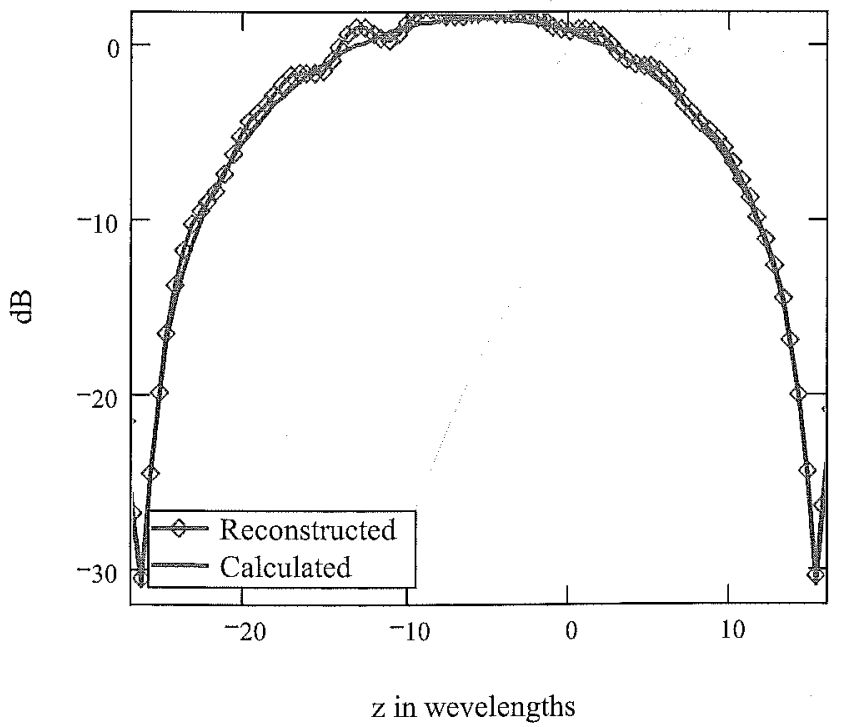

Figure 3 Reconstructed and calculated field $\left(E_{7}\right)$ with dependence of the component phi.

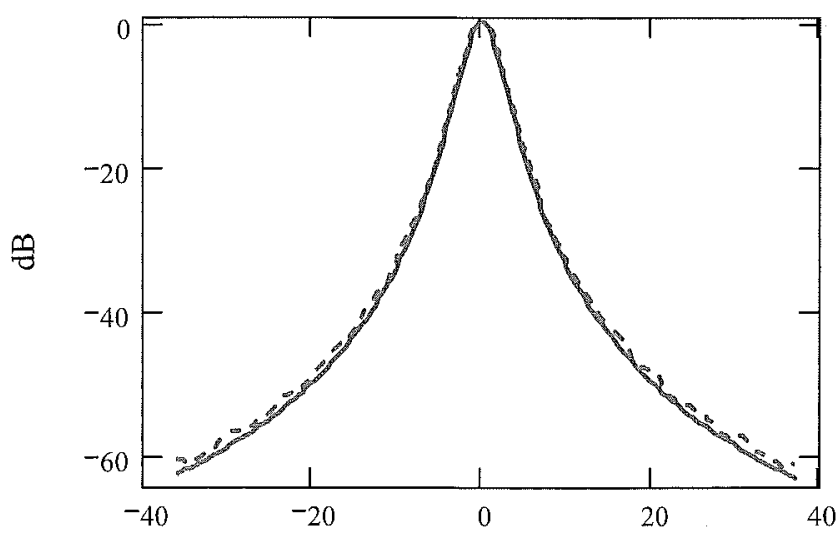

$\mathrm{z}$ in wavelengths

$$
\begin{aligned}
& \because \cdots \text { reconstructed } \\
& \text { calculated }
\end{aligned}
$$

Figure 4 Calculated and reconstructed field $\left(E_{z}\right)$ of dipole without dependence of the component phi.

reconstructed for a dipole distributed rectangular taking into account the electric field component phi. While in Figure 4 we see the near-field diagram calculated and reconstructed for a dipole distributed linearly therefore not taking into account the electric field component phi.

\section{CONCLUSION}

We have good results in the determination of the aperture field distribution of an array antenna from near-field measurement over the surface of a right circular cylinder enclosing the antenna.

The method takes advantage of the possibility, present in most of the scanning set-ups, to move the probe not only on the scanning surface, but also along the axis perpendicular to it. This possibility allows us to modify the standard cylindrical measurement procedure in order to recover the information contained in the part of the scanning surface external to the actual scanning area.

\section{REFERENCES}

O.M. Bucci and C. Gennarelli, Use of sampling expansions in nearfield-far-field transformations: The cylindrical case, IEEE Trans Antennas Propagat 36 (1988), 830-835.

W.M. Leach, Jr and D. Paris, Probe compensated near-field measurements on a cylinder, IEEE Trans Antennas Propagat AP-21 (1973), 435-445.

O.M. Bucci, A new method for avoiding the truncation error in nearfield antennas measurements, IEEE Trans Antennas Propagat 54 (2006), 2940-2951.

A. Yaghjian, An overview of near-field antenna measurements, IEEE Trans Antennas Propagat AP-34 (1986), 30-45 